\title{
Magnetic microreactors for efficient and reliable magnetic nanoparticles surface functionalization
}

\author{
R. G. Digigow, ${ }^{\mathrm{a}, \mathrm{b}}$ J-F. Dechézelles, ${ }^{\mathrm{a}} \mathrm{J}$. Kaufmann, ${ }^{\mathrm{c}}$ D. Vanhecke, ${ }^{\mathrm{a}} \mathrm{H}$. Knapp, ${ }^{\mathrm{c}} \mathrm{M}$. \\ Lattuada, ${ }^{a}$ B. Rothen-Rutishauser, ${ }^{a}$ A. Petri-Fink ${ }^{a, b} *$
}

\begin{abstract}
Microreactors have attracted wide attention in the nano- and biotechnology field because they offer many advantages over standard liquid phase reactions. We report the development of a magnetic microreactor for reliable, fast and efficient surface functionalization of superparamagnetic iron oxide nanoparticles (SPIONs). A comprehensive study is described of the development process in terms of setup, loading capacity and efficiency. We have performed experimental and computational studies in order to evaluate the trapping efficiencies, maximum loading capacity and magnetic alignment of the nanoparticles. The results show that capacity and trapping efficiencies are directly related to the flow rate, elution time and reactor type. Based on our results and the developed magnetic microreactor, we describe a model multistep surface derivatization procedure of SPIONs.
\end{abstract}

\section{Introduction}

The controlled nanoparticle surface functionalization with molecules (such as fluorescent dyes or synthetic polymers) or biomolecules (such as polysaccharides, enzymes, proteins, or peptides $)^{1}$ is a basic requirement for their use in the biomedical field in order to interact with specific targets, allow for monitoring, or to enhance colloidal stability in a biological environment. ${ }^{2,3}$ Surface functionalization strategies have been established during recent years using standard bioconjugation techniques ${ }^{4}$, supramolecular or bioorthogonal chemistry ${ }^{5}$, or nanoparticles (NPs) such as gold ${ }^{6}$, which are almost readymade for coupling procedures due to their particular chemistry. Despite significant progress in the field, surface functionalization is usually achieved in a tedious process by adapting typical synthesis parameters such as reaction time, concentration or $\mathrm{pH}$. In addition, particle surface functionalization is also time consuming due to the purification steps, which are necessary to remove un-reacted reagents and reaction side products.

However, standard techniques such as centrifugation or dialysis $^{7,1,8}$ often result in irreversible aggregation. Purification by size exclusion chromatography, which is repeated after each surface functionalization step, results in suspension dilution and eventually the loss of NPs in the column.
Superparamagnetic iron oxide NPs (SPIONs) have been intensively used for different applications such as magnetic resonance imaging ${ }^{2,9,3}$ hyperthermia ${ }^{4,10}$, targeted drug delivery systems $^{5,11}$ and nano-catalytic systems ${ }^{6,12}$. Their synthesis is well known and controlled via different synthetic pathways ${ }^{7,13}$. However, for most applications, SPIONs require quite substantial surface functionalization. Here we use magnetic immobilization in order to bypass the need for repeated purification and to allow for fast and efficient surface functionalization. Based on our previous work, we magnetically immobilized SPIONs in a microreactor using a high gradient permanent magnetic field ${ }^{14}$. Microfabricated systems for microreactions have been increasingly investigated in nanoand biotechnology because of the small volumes required ${ }^{15,16}$, the rapid heat exchange and mass transfer and the possibility to perform high-throughput experiments ${ }^{17}$. Thus, miniaturized systems have been designed for solid phase synthesis and analytical or sensing systems as they provide large surface and interface areas ${ }^{16,17}$. However, such microfluidic systems can, in analogy to our previous studies, also be employed for solid phase multistep biofunctionalization of SPIONs.

Here, we report on the design of cheap and efficient microreactors for fast and easy surface functionalization of magnetic nanoparticles. In particular, we study different microreactor setups and investigate the impact of flow rate, 
elution time and reactor type on trapping efficiency. We also propose a computational model of the trapping efficiency of the magnetic separation unit as a function of time. Our data proves how multistep SPIONs surface functionalization can easily be accomplished for future applications requiring highly complex multifunctional magnetic nanoparticles.

\section{Materials and Methods}

\section{Materials}

All chemicals were of analytical reagent grade and were used without further purification. (3-Aminopropyl)triethoxysilane (APTES, 99\%), absolute ethanol (99\%), glycerol (99.5\%), and iron (III) nitrate nonahydrate $(98 \%)$, were supplied by SigmaAldrich (Switzerland). Methanol (99\%), hydrochloric acid (37\%), isopropanol $(99.5 \%)$, potassium hexacyanoferrate (II) trihydrate $(98 \%)$ and glacial acetic acid $(99.9 \%)$ were obtained from Merck (Switzerland). Ammonia aqueous solution (28\%), nitric acid (65\%), disodium tetraborate decahydrate (98\%), and sodium hydroxide (99\%) were supplied by VWR (Switzerland). Iron (III) chloride hexahydrate (98\%), iron (II) chloride tetrahydrate $(99 \%)$, and boric acid $(98 \%)$ were purchased from Fluka (Switzerland). Epon 812 (EMBed-812), dodecenyl succinic anhydride (DDSA), (methyl-5-norbornene-2,3dicarboxylic anhydride (NMA) and 2,4,6tri(dimethylaminoethylphenol) (DMP-30) were supplied by EMS (Electron Microscopy Sciences - USA). Peptide sequence cRGD (cyclo[Arg-Gly-Asp-D-Phe-Lys(Ac-SCH2CO)]) was purchased from Peptides International (USA). Maleimide-PEGcarboxyheptyl-NHS (Mal-PEG-NHS, MW 5000) was supplied by NOF Corporation (Japan). AlexaFluor ${ }^{\circledR} 488$ Carboxylic Acid, Succinimidyl Ester, mixed isomers and 5-((2-(and-3)-S(acetylmercapto) succinoyl) amino) Fluorescein, mixed isomers (SAMSA Fluorescein), were purchased from Invitrogen (Switzerland).

Dialysis membrane tubing with a molecular weight cutoff of 12 $\mathrm{kDa}$ was used for purification (D-9527 Sigma, cellulose membrane). All aqueous solutions were prepared with ultrapure Milli-Q water (18.2 $\mathrm{m} \Omega$, Millipore AG).
The FeNdB magnets were purchased from Maurer Magnets (Switzerland - M662056, 20*10*5 mm, 1* $\mathrm{W}^{*} \mathrm{~h}$ ) and nickel mesh (nickel gauze, 100 meshes woven with $0.1 \mathrm{~mm}$ diameter wire, mesh width $=0.2 \mathrm{~mm}$ ) was supplied by Alfa Aesar (Switzerland). Two types of nickel foam (50 pores per inches (PPI) and 100 PPI, 96 - $98 \%$ porosity) were supplied by American Elements (USA). An HPLC Pump 64 supplied by Knauer AG (Switzerland) and an ultrasonic tube transducer (Probe - TE-20-11433, auto frequency power) from Telsonic Ultrasonics (Switzerland) were used for sample preparation and dispersion.

\section{Microreactor Design and Development}

The here-described microreactor was developed for research laboratories working with magnetic nanoparticles for easy, efficient and reproducible particle surface functionalization. The reactor geometry design is driven by different requirements, such as efficient particle retention, elution and easy priming. Nickel is often used to produce discontinuity in the magnetic permeability, because it can be strongly magnetized and shows at the same time low magnetic remanence ${ }^{18}$. The reactor should be as thin as possible in order to minimize the distance between the nickel discontinuity (i.e. either nickel mesh or nickel foam) and the permanent magnets for maximum magnetic field strength and particle retention. In order to facilitate particle elution, smaller cross-sections result in higher flow velocities and thus higher shear stresses at constant volume flows ${ }^{19,20}$. The outer dimensions of all microreactors were $24 \times 13 \times 3 \mathrm{~mm}(1 \mathrm{x} \quad \mathrm{w} \times \mathrm{x})$; the inner dimensions of the nickel mesh and nickel foam based reactors varied between $7 / 2.2 \mathrm{~mm}$ (inner width/inner height) for the nickel mesh based reactor and $9 / 1.6 \mathrm{~mm}$ for the nickel foam based reactor, respectively. All microreactors were made from polystyrene, which is cheap and easy to process and nonreactive with respect to the aqueous suspensions used in the reactor. Figure 1 shows microreactor type A, which refers to the nickel mesh based microreactor, the position of the permanent magnets and the nickel mesh, in addition to the particle alignment in the reactor.
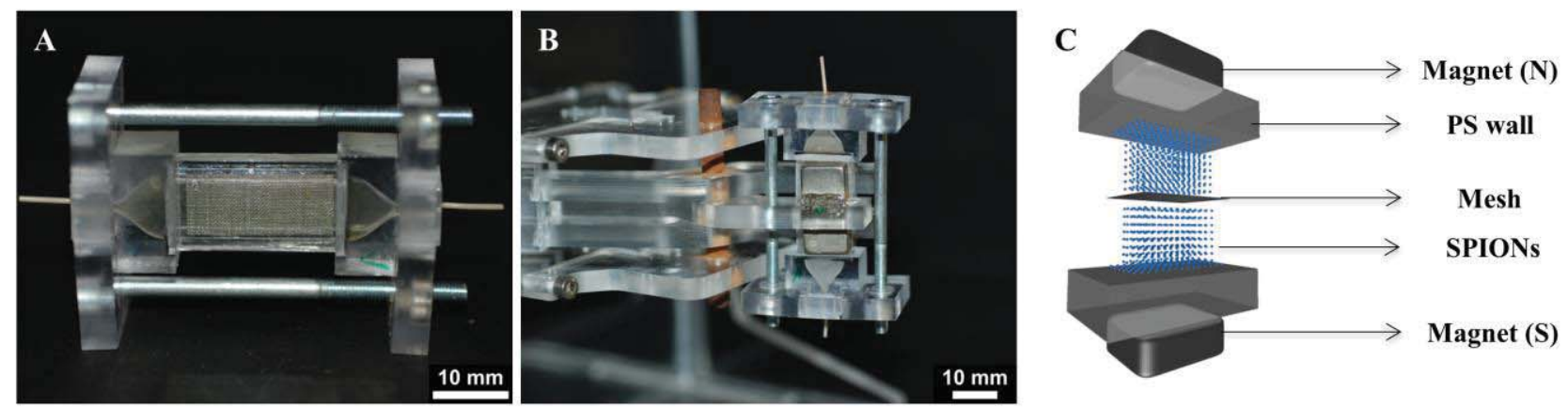

Figure 1: Images of the (A) magnetic microreactor without magnets and (B) full set-up including the reactor holder, the magnetic microreactor, and the permanent magnets. (C) Schematic illustration of the nanoparticle alignment (blue dots) in the reactor chamber. 


\section{Particle syntheses and characterizations}

\section{Synthesis AND Surface Modification OF SPIONs}

SPIONs were synthesized by the alkaline co-precipitation of ferric and ferrous chlorides in aqueous solution ${ }^{21}$ following the work reported by $\mathrm{Bee}^{22}$ and van Ewijk ${ }^{23}$. Surface modification of SPIONs with APTES was accomplished adapting the procedure of Yamaura et al. ${ }^{24}$. Briefly, $5 \mathrm{~mL}$ of SPIONs $(10 \mathrm{mg}$ iron/mL) were mixed with $5 \mathrm{~mL}$ of glycerol and $45 \mathrm{~mL}$ of methanol in a three-neck round bottom flask. The mixture was heated to $85^{\circ} \mathrm{C}$ under stirring $(750 \mathrm{rpm})$ and $10 \mathrm{~mL}$ of aqueous APTES solution (10\% v/v, $\mathrm{pH}=4$ adjusted with acetic acid) was added slowly to the particle suspension using a dropping funnel. After three hours, the suspension was cooled down to room temperature and centrifuged at $30,000 \mathrm{~g}$ for 45 minutes. The supernatant was discarded and the pellet redispersed in 100 $\mathrm{mL}$ of Milli-Q water. This purification step was repeated twice and the final suspension of APTES-coated SPIONs was stored at $4^{\circ} \mathrm{C}$.

\section{Surface PEGylation AND COUPLING OF A SMALL PEPTIDE}

Amine functional silica coated iron oxide beads ${ }^{25}$ were surface functionalized using Maleimide-Polyethylene glycolcarboxyheptyl-NHS (Mal-PEG-NHS) in the magnetic microreactor. Thereafter, $0.5 \mathrm{mg}$ immobilized magnetic beads were PEGylated by recirculating $5 \mathrm{ml}$ Mal-PEG-NHS (1.25 $\mathrm{mg} / \mathrm{mL}$ Mal-PEG-NHS in $20 \mathrm{mM}$ borate buffer solution, $\mathrm{pH}$ 7.5 ) at a flow rate of $0.5 \mathrm{ml} / \mathrm{min}$ for 60 minutes. After the reaction, the PEGylated nanoparticles were continuously washed with $5 \mathrm{ml}$ of $20 \mathrm{mM}$ borate buffer.

To allow for fluorescent detection and quantification, amine functional silica coated iron oxide beads were also PEGylated using a dye-labeled Maleimide-Polyethylene glycolcarboxyheptyl-NHS (Mal-PEG-NHS) in the magnetic microreactor. Prior to functionalization, $0.6 \mathrm{~mL}$ of the fluorescent dye $(8.34 \mathrm{mg} / \mathrm{mL}$ - SAMSA Fluorescein) was reacted with $5 \mathrm{ml}$ Mal-PEG-NHS $(1.25 \mathrm{mg} / \mathrm{mL}$ Mal-PEG-NHS in $20 \mathrm{mM}$ borate buffer solution, $\mathrm{pH}$ 7.5) at room temperature. The reaction occurred between thiol group of the fluorescent dye and the maleimide group of the heterobifunctional PEG via maleimidesulfhydryl chemistry.

Thereafter, $0.5 \mathrm{mg}$ of immobilized magnetic beads were PEGylated by recirculating $5 \mathrm{ml}$ Dye-PEG-NHS $(1.25 \mathrm{mg} / \mathrm{mL}$ in $20 \mathrm{mM}$ borate buffer solution, $\mathrm{pH} 7.5$ ) at a flow rate of 0.5 $\mathrm{ml} / \mathrm{min}$ for 60 minutes. After the reaction, the dye-PEGylated particles were continuously washed with $5 \mathrm{ml}$ of $20 \mathrm{mM}$ borate buffer $(\mathrm{pH}=6.8)$, eluted from the reactor and re-dispersed in phosphate buffered saline (PBS).

Prior to functionalization, $1 \mathrm{mg}$ of cRGD (Arg-Gly-Asp-D-PheLys (Ac-SCH2CO)) was de-protected using $10 \mu \mathrm{l}$ of $0.5 \mathrm{M}$ sodium methoxyde and diluted in borate buffer. A total of 10 $\mu \mathrm{L}$ of the cRGD peptide solution was mixed with $0.5 \mathrm{~mL}$ AlexaFluor ${ }^{\circledR} 488(1 \mathrm{mg} / \mathrm{mL})$ and allowed to react at room temperature for 1 hour. The product cRGD-AlexaFluor®488 was diluted in $5 \mathrm{~mL}$ borate buffer $(20 \mathrm{mM}, \mathrm{pH}$ 6.8) to a final concentration of $0.2 \mathrm{mg} / \mathrm{mL}$. To accomplish cRGD coupling, 1 $\mathrm{ml}$ of the cRGD-AlexaFluor ${ }^{\circledR 488}$ solution was added to the immobilized PEGylated beads in the microreactor and allowed to react. The obtained cRGD-PEG-SPIONs were purified again with $5 \mathrm{ml}$ of $20 \mathrm{mM}$ borate buffer and unreacted maleimide groups were quenched by adding $1 \mathrm{~mL}$ of $4 \mathrm{mg} / \mathrm{mL}$ cysteine solution. The functionalized nanoparticles were subjected to another purification step, eluted from the reactor and redispersed in phosphate buffered saline (PBS).

\section{TranSMisSion ElECtron MicROSCOPY}

Particle size and morphology were studied by transmission electron microscopy (TEM). Samples were diluted in Milli-Q water (1:10) and one drop of the diluted suspension was slowly evaporated on a 300 mesh carbon membrane-coated copper grid. TEM experiments were performed on a Philips CM100 Biotwin microscope operated at $80 \mathrm{kV}$ and a FEI Morgani operated at $80 \mathrm{kV}$.

\section{Prussian Blue Colorimetric AsSAy}

The iron content of the suspensions was determined by the Prussian blue assay ${ }^{26}$. Thereafter, $50 \mu \mathrm{L}$ of APTES-coated SPIONs (0.020 to $0.100 \mathrm{mg}$ of iron / $\mathrm{mL}$ ) were dissolved in 100 $\mu \mathrm{L}$ hydrochloric acid $(6 \mathrm{M})$ in a $96-w e l l$ plate. The solution was mixed with $100 \mu \mathrm{L}$ of a $5 \%$ aqueous solution of potassium hexacyanoferrate (II) trihydrate and the absorbance was read at $690 \mathrm{~nm}$ after 10 minutes in a multi-well plate reader (Victor3 Perkin Elmer).

\section{Size And Zeta Potential}

Dynamic light scattering (DLS) measurements were carried out at fixed angle $\left(90^{\circ}\right)$ on a photon correlation spectrometer from Brookhaven equipped with a BI-9000AT digital autocorrelation. The nanoparticles were redispersed from the original suspension in $20 \mathrm{mM}$ borate buffer $(\mathrm{pH}=7.5)$ and the concentrations were set between 0.030 to $0.100 \mathrm{mg}$ of iron / $\mathrm{mL}$ to avoid multiple light scattering.

Zeta potential measurements were performed on a Particle Size Analyzer 90 plus from Brookhaven Instruments equipped with a BI-9000AT platinum electrode. Viscosity, refractive index, and the dielectric constant of pure water at $25^{\circ} \mathrm{C}$ were used. The electrode was cleaned for 5 minutes in an ultrasonic bath prior to each measurement and pre-equilibrated for two minutes in small volume of the sample. The samples were prepared accordingly for DLS measurements.

\section{MAGNetic PRoperties}

A vibrating-sample magnetometer (VSM, Princeton Measurements Corporation Vibrating Sample Magnetometer model 3900) was used at room temperature to study the magnetic properties of APTES-coated SPIONs. Magnetization was measured as a function of the applied field up to 796 $\mathrm{kA} \cdot \mathrm{m}^{-1}$, using an averaging time of $100 \mathrm{~ms}$. 


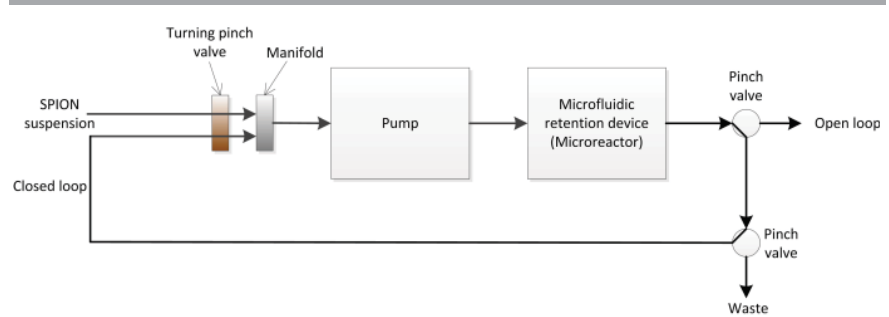

Figure 2: Schematic illustration of the microreactor system with the two possible configurations (closed or open loop).

\section{FLUORIMETRIC ASSAY}

The number of fluorescent molecules per particle was determined by a fluorometric assay. Standard curves were generated using SAMSA Fluorescein $(0.1$ to $1.0 \mu \mathrm{g} / \mathrm{mL}$, in Milli-Q water, $50 \mu \mathrm{L}$ ). The fluorescence intensity was read at an emission wavelength of $520 \mathrm{~nm}$ in a multi-well plate reader $\left(\lambda_{\text {exc }}=496 \mathrm{~nm}\right.$, Victor3 plate reader, Perkin Elmer, USA). The concentration of the fluorescent dye in the sample was estimated with the calibration curve obtained from standards. The amount of dye per particle was calculated using the hydrodynamic diameter obtained by DLS.

\section{FLUORESCENCE SPECTRA}

Fluorescence spectra of particle suspensions were measured in a quartz cuvette $(10 \mathrm{~mm})$ with a Photon Technology International C720 spectrophotometer equipped with a Hamamatsu R928P photomultiplier. Spectra were acquired in $0.1 \mathrm{sec}$ from 500 to $600 \mathrm{~nm}$ at $\lambda_{\mathrm{exc}}=490 \mathrm{~nm}$.

\section{Microreactor setup}

As illustrated in figure 2, the microreactor was inserted into a loop system composed of an HPLC pump and a valve to switch from an open to a closed loop setup. Two permanent magnets were used for magnetic immobilization of the NPs in the microreactor chamber.

Open and closed loop experiments were carried out using APTES-coated SPIONs as model nanoparticles to study trapping efficiency and elution capacity of the different microreactors. The scheme of both experiments is illustrated in figure 2. Open loop experiments, (i.e. the particle suspension was introduced once without recirculation) were carried out to study nanoparticle immobilization and elution as a function of time while closed loop experiments, (i.e. nanoparticle suspension was recirculated) were performed to evaluate the trapping efficiency of each version of the microreactor.

\section{OPEN LOOP SETUP}

Nanoparticle immobilization and elution were studied over time by measuring the iron concentration of samples that were regularly collected (every minute) during loading and the elution process. Thereafter, APTES-coated SPIONs were loaded into the microreactor and circulated in the open loop system (Figure 2). The microreactor was connected to an HPLC pump using Teflon tubes and the system was washed by circulating Milli-Q water for 15 minutes $(0.5 \mathrm{~mL} /$ minute). The reactor chamber was placed between two magnets (attractive mode). To start the experiment, Milli-Q water was circulated in the system at $0.5 \mathrm{~mL} /$ minute for 5 minutes. Then, the suspension was loaded and circulated in the system at the same flow rate for 20 minutes. Untrapped nanoparticles were removed by washing with Milli-Q water for 5 minutes. To study particle elution the magnets were removed and Milli-Q water was circulated again for 15 minutes. Ultrasound was applied to the reactor chamber with an ultrasonic tube transducer to improve particle elution. Three high frequency ultrasound treatments were applied for 30 seconds, separated by 1 minute. The iron concentration of the collected samples was determined by the Prussian blue assay.

\section{Closed Loop Setup}

APTES-coated SPIONs were loaded and circulated in the microreactor in a closed loop setup (Figure 2) to study and compare particle-trapping efficiencies. The system was designed as described above for the open loop system but instead, the particles were circulated for different time periods in the closed loop system $(0.5,2,6$ and 24 hours) using different flow rates $(0.1,0.5$ and $1.0 \mathrm{~mL} / \mathrm{min})$. Afterwards, untrapped nanoparticles were independently collected to determine the iron concentration by the Prussian blue assay.

\section{Particle Alignment}

In order to visualize the magnetic alignment of the APTEScoated SPIONs in the microreactor, the suspension was introduced and magnetically immobilized in the microreactor (type A). The microreactor was cleaned by circulation of Milli$\mathrm{Q}$ water and absolute ethanol $(0.5 \mathrm{~mL} /$ minute $)$ for 10 minutes and 15 minutes, respectively. Then, $10 \mathrm{~mL}$ APTES-coated SPIONs $(0.2 \mathrm{mg}$ of iron / $\mathrm{mL})$ were loaded in the closed loop system for 1 hour. After the nanoparticles had been fully immobilized, an epoxy resin (Epon, Electron Microscopy Sciences - USA) was carefully introduced into the reactor. The epoxy resin was prepared by mixing $5 \mathrm{~mL}$ of EMBed-812 with $8 \mathrm{~mL}$ of DDSA (mixture 1). $8 \mathrm{~mL}$ of EMBed-812 were mixed with $7 \mathrm{~mL}$ of NMA (mixture 2). Finally, $6.5 \mathrm{~mL}$ of mixture 1 were slowly mixed with $7.5 \mathrm{~mL}$ of mixture 2 . Afterwards 0.225 $\mathrm{mL}$ of the initiator DMP-30 were added and stirred very carefully for 5 minutes to activate the polymerization of the resin. The mixture was heated to $60^{\circ} \mathrm{C}$ to reduce the viscosity and was slowly introduced in the microreactor $(0.05 \mathrm{~mL} /$ minute). After the entire system had been completely filled with the epoxy resin, the pump was stopped and the microreactor was kept at $60^{\circ} \mathrm{C}$ for 24 hours. The magnets were removed and the resin block containing the immobilized APTES-coated SPIONs was cut perpendicular to the magnets, i.e. from the mesh to the reactor chamber wall, in $250 \mathrm{~nm}$ thick slices using a ultramicrotome (Leica, Austria) equipped with a diamond knife (Diatome, Switzerland). The slices were placed on a 300 mesh carbon membrane-coated Quantifoil R2/1 copper grid and observed in a Tecnai F20 TEM (FEI, USA). 

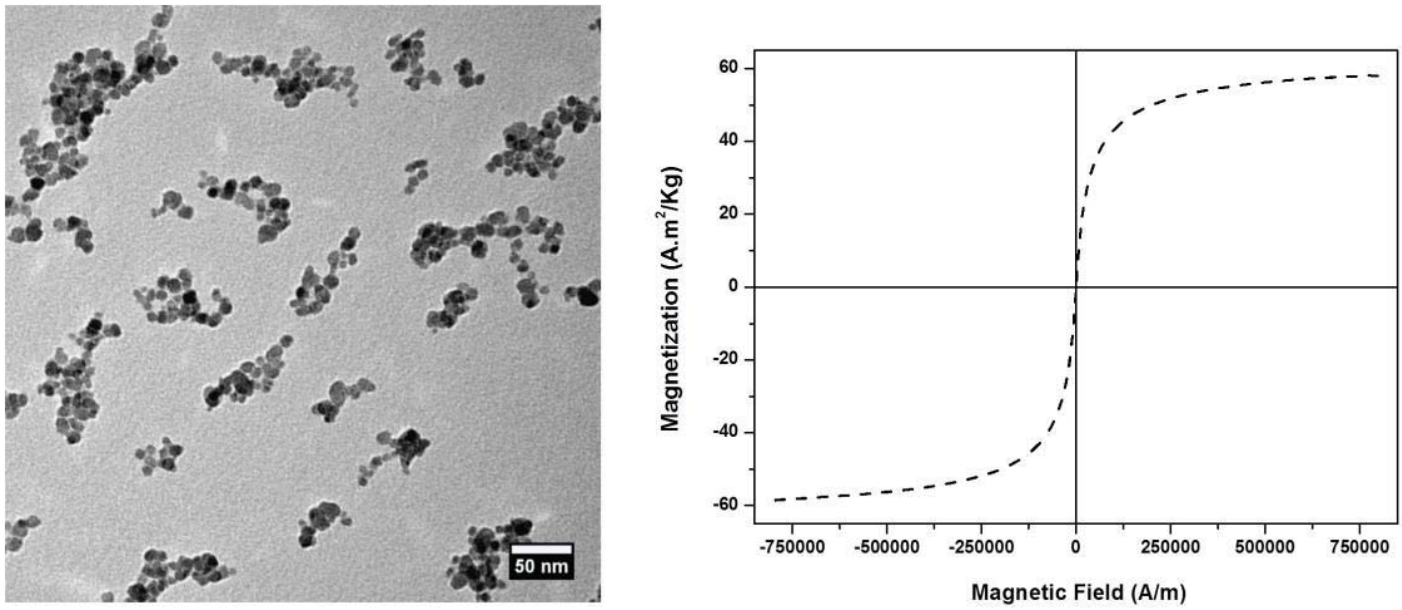

Figure 3: Transmission electron micrograph and magnetization curve of the APTES-coated SPIONs.
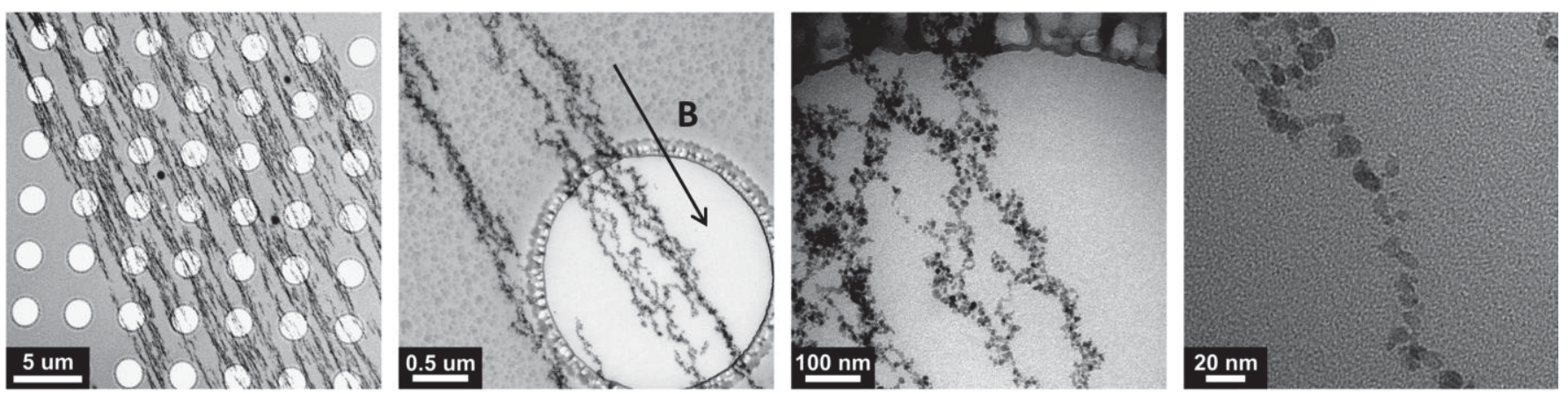

Figure 4: TEM images of the magnetic alignment (B: magnetic field direction) of the NPs inside the microreactor (from left to right: increasing magnification). The regular perforations are typical for the Quantifoil support film.

\section{Simulations}

All ordinary differential equations in the model have been solved with Matlab (Mathworks, version 2013a) using ODE15s as differential equation solver. The two-dimensional convection-diffusion partial differential equation used to estimate the rate of particle trapping on a wire was solved using a finite difference code programmed in FORTRAN (Intel Fortran Parallel Studio 2011). A fourth order implicit finite difference algorithm with implicit time stepping was used. A very fine spatial grid was utilized, with up to 400,000 grid points. In order to deal with the large memory occupancy resulting from the discretization, a sparse matrix approach has been used to store the coefficients of the discretized equation.

\section{Results and discussion}

\section{Nanoparticles characterization}

The synthesized SPIONs were coated with APTES to provide free amine groups for further surface functionalization, such as the coupling of antibodies, short peptides, or fluorescent dyes. Figure 3 shows a transmission electron micrograph of APTEScoated SPIONs. The increased polydispersity and average size of $47 \pm 22 \mathrm{~nm}$ of APTES-coated SPIONs can be explained by the formation of aggregates in water, which was previously attributed to the hydrophobicity of the organosilane ${ }^{27,28}$. Also, APTES coating induces a decrease of the surface potential because it reduces the possibility of adsorption of potentialdetermining ions on the surface. As the charge diminishes, the electric forces of repulsion between the nanoparticles weakens and consequently, the nanoparticles aggregate ${ }^{27}$.

Zeta potential and particle size were measured before trapping and after particle elution. The nanoparticles displayed a constant zeta potential of 25.1, 25.0 and $27.2 \mathrm{mV}$ after trapping and elution from all three microreactors A, B and B' respectively. Dynamic light scattering showed a slight increase of particle hydrodynamic diameters from $47 \pm 22 \mathrm{~nm}$ to $64.6 \pm$ $29.2 \mathrm{~nm}, 61.7 \pm 27.3 \mathrm{~nm}$ and $54.4 \pm 23.5 \mathrm{~nm}$, for NPs trapped in microreactors A, B and B' respectively. This size increase might be attributed to the presence of larger aggregates, which were formed in the microreactor chamber ${ }^{29}$.

Magnetization of APTES-coated SPIONs was measured as function of the magnetic field using a VSM at room temperature. Figure 3 and SI-6 show the typical magnetization curves of a superparamagnetic material ${ }^{30}$. 

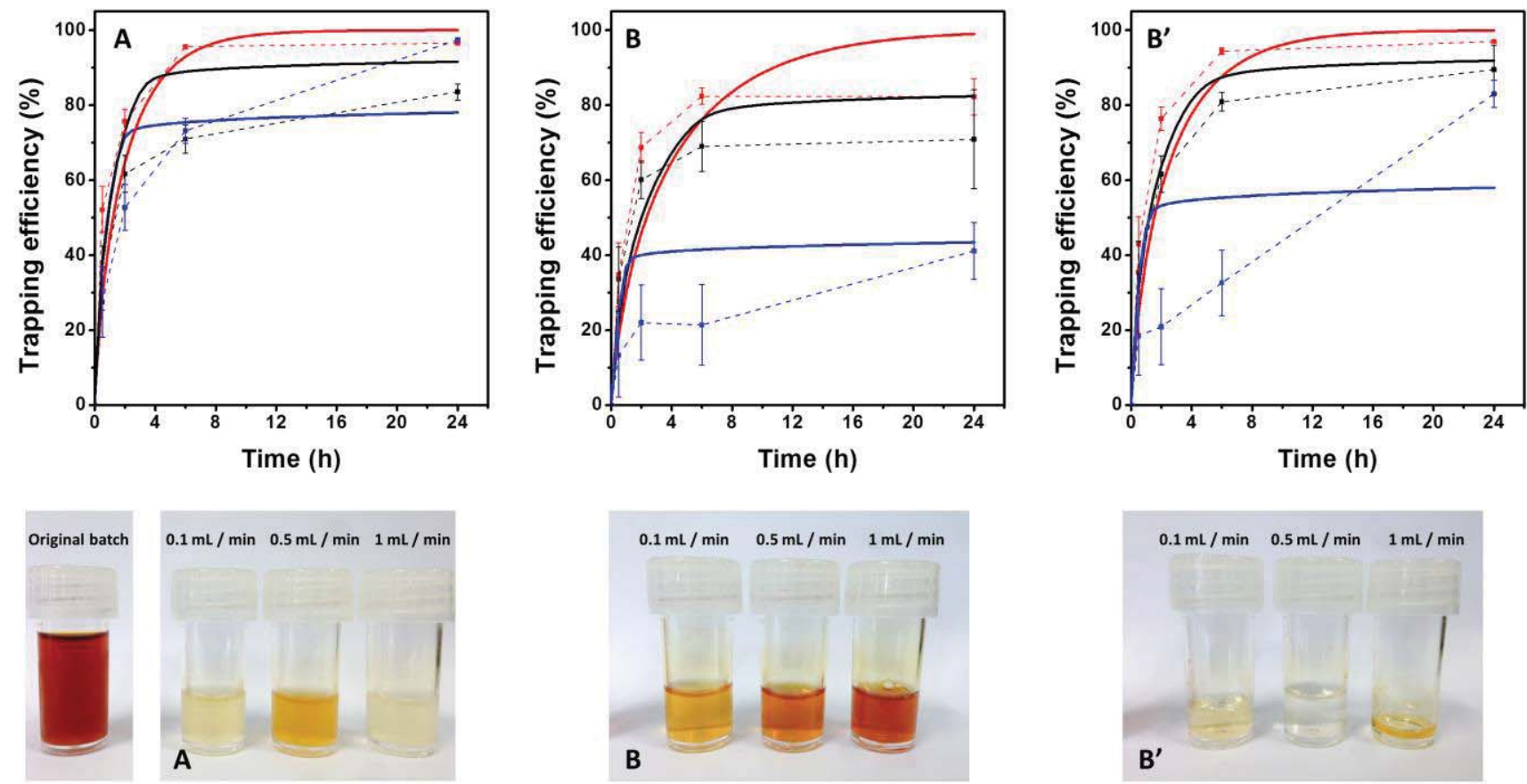

Figure 5: Top: Time dependent trapping efficiency for each microreactor type (A, B, and $\left.\mathrm{B}^{\prime}\right)$ as a function of the flow rate $(0.1 \mathrm{~mL} / \mathrm{min}$ (red), $0.5 \mathrm{~mL} / \mathrm{min}(\mathrm{black})$, and 1 $\mathrm{mL} / \mathrm{min}$ (blue). Experimental data (dotted line) and computational simulation (solid lines). Bottom: Pictures of the original APTES-coated SPIONs (left) and suspensions of untrapped nanoparticles after 24 hours circulation at $0.1,0.5$ and $1 \mathrm{~mL} / \mathrm{min}$.

\section{Nanoparticles alignment}

In order to visualize particles alignment in the microreactor, an epoxy resin was loaded to immobilize the trapped NPs in the microreactor chamber. This resin was polymerized in situ in the chamber and was cut into slices for TEM characterization after drying (Figure 4). As expected, the NPs align in long chains from the PS wall close to the magnet to the nickel mesh, along the direction of the applied magnetic field (Figure SI-1) ${ }^{31}$. Individual NPs and small particle clusters can be detected at higher magnification (Figure 4).

\section{Trapping efficiency}

Figure 5 shows the impact of flow rate and reactor setup on the trapping efficiency of the three different reactors type over time. The experiments were performed by introducing $5 \mathrm{~mL}$ of nanoparticle suspension $(605 \mu \mathrm{g}$ of iron $/ \mathrm{mL})$ in the microreactor in its closed loop configuration. The trapping efficiency was calculated as:

$$
\text { Trapping efficiency }(\%)=\frac{\text { total amount of loaded iron }- \text { amount of untrapped iron }}{\text { total amount of loaded iron }} \times 100
$$

The color of the brownish suspension changed in all experiments over time, thus indicating particle trapping in the microreactors (Figure 5, bottom). Beyond this qualitative aspect, the evolution of the trapped iron mass as well as the particle trapping efficiency for each microreactor type was investigated.

Figure 5 (top) shows the trapping efficiency of the different reactors over time and as a function of the flow rate. All reactors showed increasing trapping efficiencies with time. Three flow rates $(0.1,0.5$ and $1 \mathrm{~mL} / \mathrm{min})$ were investigated to evaluate their impact on particle trapping. Experiments carried out at $0.1 \mathrm{~mL} / \mathrm{min}$ always showed the highest trapping efficiency for all reactor types and time points measured, reaching $96.5 \%, 83.5 \%$ and $97.4 \%$ after $24 \mathrm{hrs}$ for reactor types A, B, and B', respectively (Table SI-1). In addition, trapping saturation was reached much faster at $0.1 \mathrm{~mL} / \mathrm{min}$ (Figure 5, top). In general, higher flow rates (i.e. $0.5 \mathrm{~mL} / \mathrm{min}$ and $1 \mathrm{~mL} / \mathrm{min}$ ) resulted in lower overall trapping efficiency at early time points. Larger volumes resulted in significantly reduced capturing efficiency (Table SI-1). The amount of captured NPs is determined by different parameters, such as the competition between magnetic force of the magnets and hydrodynamic force of the circulating suspension ${ }^{32}$, the number of cycles $^{33}$, the suspension volume, and the nature of the nickel support, i.e. nickel mesh vs. nickel foam. Since the magnetic force of each microreactor is the same $(0.5 \mathrm{~T})$, the flow rate is the only modified parameter when comparing microreactors A, $\mathrm{B}$ and $\mathrm{B}$ '.

Microreactor A contains a nickel mesh composed of individual nickel wires in the center of the reactor chamber. Even at high flow rates, the NPs can move freely in the reactor. The chambers of microreactors B and B' are filled with the nickel foam (50 and 100 PPI (pore per inch), respectively). The high number of passages through the magnetic chamber might result in the formation of aggregates in the suspension, which can be due to the magnetic field. It is known that aggregation results from magnetic interactions between NPs in the presence of external magnetic fields ${ }^{29}$. Thus, the small pore sizes of in 
particular the nickel foam microreactor B' can effectively prevent nanoparticle circulation thereby inducing the capture of large SPIONs aggregates and consequently higher iron amounts. Moreover, when the pores are small enough (microreactor $\mathrm{B}^{\prime}$ ), this barrier can reduce the velocity of the particle suspension within the reaction chamber, which consequently reduces the effect of the hydrodynamic force ${ }^{34}$. In accordance and independent of the flow rate, microreactor $\mathrm{B}$ was less efficient than microreactors A and B', which was also confirmed by the darker and more intensive color of the suspensions (Figure 5, bottom). A particularly high trapping efficiency was observed in the experiment with microreactor A at $1 \mathrm{~mL} / \mathrm{min}$. This could be explained by the higher number of passages through the magnetic field resulting in the formation of bigger aggregates.

\section{Simulation}

Simulation of magnetic separation units has a long history $^{20,33,35-40}$. A great deal of modeling work has been carried out in the eighties and in the nineties, with the objective to quantify the performances of high gradient magnetic separation (HGMS) units, as they are commonly referred to. Most of the work has focused on predicting the amount of NPs that can be trapped in a HGMS unit at steady states under given conditions $^{20,38}$. However, for this work a step forward needs to be made, since the time dependent performance of the microreactor needs to be evaluated, both over a short time and a long time.

The model adopted involves a multiscale approach. Macroscopically, two mass balance equations are solved, the first one to follow the time evolution of the concentration of magnetic NPs eluting through the column, the second one quantifying the amount of NPs accumulating on the wires mesh (or on the foam). In most of the models adopted in the literature, these equations were partial differential equations, allowing monitoring the time evolution of particle concentration as a function of time and position along the column. However, the short length of the microreactors developed in this work compared to their cross-section permits to considerably simplify the problem by modeling them as perfectly mixed systems with a uniform concentration of NPs and reduce the problem to ordinary differential equations. The first balance equation can be written as follows ${ }^{36,38}$ :

$$
\frac{d n}{d t}=\frac{Q}{V}\left(n_{0}-n\right)-K \cdot n
$$

Where $n$ is nanoparticle concentration inside the microreactor not bound to mesh, $n_{0}$ is the concentration of NPs in suspension entering the microreactor, $Q$ is the flow rate of the suspension going through the microreactor, $V$ is the reactor volume and $K$ is the trapping rate of NPs per unit volume. Equation (1) simply states that the accumulation (or depletion) of NPs in the reactor is given by the balance between trapping rate of NPs on the mesh and the difference of loaded and removed NPs from the microreactor by convection. The second equation is a balance equation for the NPs immobilized onto the wire mesh $^{38}$ :

$$
\frac{d n_{B}}{d t}=K \cdot n
$$

Where $n_{B}$ is the concentration of NPs per unit volume in the socalled buildup, i.e. the NPs trapped on the wire mesh. The trapping rate $K$ of NPs contains all the information about the physics of the retention process. In the case of loop experiments, a third differential equation is added to account for the mass balance of NPs fed to - and recycled from - the microreactor. Once again, it is assumed that the loop is obtained by connecting the microreactor to another perfectly mixed reactor, which simply feeds NPs at a concentration $n_{0}$ and receives NPs from- the microreactor at a concentration $n$. The mass balance over this perfectly mixed reactor is then given by:

$$
\frac{d n_{0}}{d t}=\frac{Q}{V_{L}}\left(n-n_{0}\right)
$$

In Equation (3) $V_{L}$ is the volume of the particle suspension used in the loop experiments.

In order to solve Equations (1-3), the rate of NPs trapping by the wires $K$ is required, as well as its dependence on the operating conditions, such as magnetic field strength used, flow rate and particle concentration, The retention of NPs by a magnetic wire results from the competition of three mechanisms: magnetic interactions, diffusion and convection 20,33,39,40. Magnetic interactions are responsible for the accumulation of NPs around a wire, creating strong concentration gradients of NPs, thus leading to high diffusion fluxes that tend to counterbalance magnetic interactions. In the absence of convection, at steady state, diffusion and magnetic interaction counterbalance each other. The equilibrium profile defines the maximum amount of NPs per unit length of wire that can be accumulated. The equilibrium is more complex in the presence of convection. A comprehensive description of the accumulation kinetics of NPs around an isolated wire requires the solution of a two-dimensional convection-diffusion equation including all the above mentioned mechanisms ${ }^{39,40}$. The detailed equation is reported in the Supplementary Information. Such a solution has been carried out only for a few conditions using a finite difference method ${ }^{39}$. The objective of solving such equation was to find an angular average expression for both the velocity profile and the magnetic interaction potential. The equilibrium concentration profile of NPs around a wire can be found by solving the following one dimensional non-linear differential equation ${ }^{41,42}$ :

$$
\frac{1}{r} \frac{d}{d r}\left(r\left(D(\phi) \frac{d \Pi}{d \phi} \frac{\partial n}{\partial r}+\left(\frac{D(\phi)}{k T} \frac{d U_{M}}{d r}-v_{r}\right) n\right)\right)=0
$$



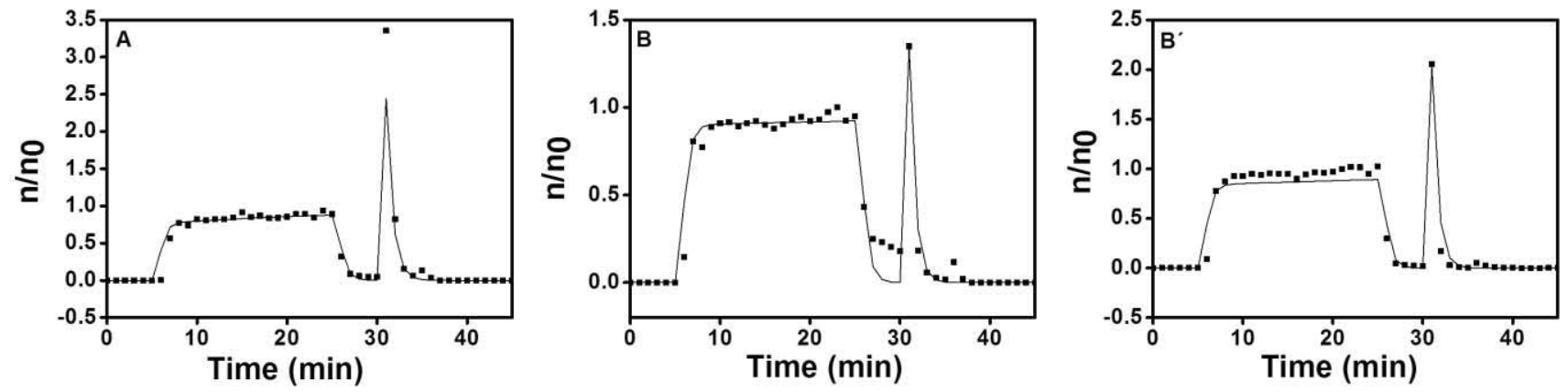

Figure 6: Computation simulation (solid lines) and experimental data (data points) of the immobilization and elution of APTES-coated SPIONs as function of time for each microreactor type (A, B, and $\left.B^{\prime}\right)$. Experiments performed in open-loop system with flow rate of $0.5 \mathrm{~mL} / \mathrm{min}$.

Here $n$ is the particle concentration, $\phi$ the particle volume fraction, $U_{M}$ the angular average magnetic interaction potential energy, $D(\phi)$ the volume fraction dependent particle diffusion coefficient, $r$ the distance from the center of the wire, $\Pi$ the osmotic pressure and $v_{r}$ the angular average convective velocity. The osmotic pressure term is necessary to prevent NPs from accumulating beyond physical limits. The expression used here is the one given by the Carnahan Starling equation ${ }^{42}$ :

$$
\Pi=\phi \frac{1+\phi+\phi^{2}-\phi^{3}}{(1-\phi)^{3}}
$$

The dependence of the diffusion coefficient on the particle volume fraction is given by the following semi-empirical equation $^{42}$ :

$$
D(\phi)=D_{0}(1-\phi)^{6.55}
$$

where $D_{0}$ is the particle diffusion coefficient at infinite dilution, which is assumed to be given by the Stokes-Einstein equation. The angular average magnetic interaction potential between a particle and a magnetized wire is defined $\mathrm{as}^{43}$ :

$\frac{U_{M}(r)}{k T}=-\log \left(\frac{1}{2 \pi} \int_{0}^{2 \pi} \exp \left(\frac{2 \pi \mu_{0} M_{p} M_{w} R_{p}^{3} R_{w}^{2}}{3 r^{2} k T}\left(\frac{M_{w} R_{w}^{2}}{4 H_{0} r^{2}}+\cos (2 \theta)\right)\right) d \theta\right)$

where $M_{w}$ is the wire magnetization, $M_{p}$ the particle magnetization, $R_{p}$ the particle radius, $R_{w}$ the wire radius, $H_{0}$ is the external applied magnetic field, assumed to be constant, $\mu_{0}$ is the magnetic permittivity of vacuum, $T$ is the absolute temperature and $k$ the Boltzmann constant. This expression accounts for the magnetic field generated by a wire with a uniform magnetization. The angular average convective velocity profile, instead, is defined using a semi-empirical formula, verified by fitting the results of the two dimensional simulations:

$$
v_{r}=\frac{\mathrm{V}_{0}}{2-\log (\mathrm{Re})}\left(\frac{\beta}{\log (r)-0.5 \cdot\left(1-\frac{1}{r^{2}}\right)}+\alpha r^{n}\right)
$$

Where $V_{O}$ is the average velocity in the microreactor, $R e$ is the wire Reynolds number $\left(R e=\rho V_{0} R_{w} / \eta\right.$, where $\rho$ and $\eta$ are the density and viscosity of water, respectively), and $\alpha$ and $\beta$ are fitting parameters. It was found that a power law dependence of these constants on Peclet number $\left(P e=V_{0} \cdot R_{w} / D_{0}\right)$ was the best option. It is further assumed that the accumulation of NPs around a wire is radially symmetric.

The integration of Equation (4) subject to the boundary conditions (the overall flux is zero at the surface of the wire, and that the concentration of NPs equals the bulk one at infinite distance from the wire surface) gives the dependence of the accumulated amount of NPs on the distance from the wire surface. An almost analytical solution of Equation (4) can be obtained. In order to calculate the rate of accumulation of NPs, it is assumed that the NPs accumulate in a layer-by-layer fashion around the wire. Consequently, the accumulation rate is given by the sum of the magnetic and convective fluxes computed at distance from the wire surface where the amount of nanoparticles accumulated around the wire is equal to a given value. The quantitative details are reported in the table 1. In summary, Equation (4) is solved first independently in order to obtain an expression for $\mathrm{K}$, which is then used in Equations (1) and (2).

The results of the simulations are shown in figures 5 and 6 . Figure 6 shows the simulation of the short-term batch experiments (open loop setup). The agreement between simulations and experiments is very satisfactory in all cases. The model can well predict both the rate at which NPs accumulate around wires, as well as the elution phase when the magnetic field is turned off. 
Table 1: Values of the parameters used in the calculations

\begin{tabular}{|c|c|}
\hline Particle radius, $R_{p}$ & $13.2 \mathrm{~nm}$ \\
\hline Mesh wire radius, $R_{w}$ & $50 \mathrm{~mm}$ \\
\hline Foam B wire radius, $R_{w}$ & $100 \mathrm{~mm}$ \\
\hline Foam B' wire radius, $R_{w}$ & $75 \mathrm{~mm}$ \\
\hline Water viscosity, $h$ & $0.000879 \mathrm{~Pa} \cdot \mathrm{s}$ \\
\hline Temperature, $T$ & $298 \mathrm{~K}$ \\
\hline Particle magnetization, $M_{p}$ & $2.66 \cdot 10^{5}[\mathrm{~A} / \mathrm{m}]$ \\
\hline Nickel wire magnetization, $M_{w}$ & $5.22 \cdot 10^{5}[\mathrm{~A} / \mathrm{m}]$ \\
\hline Particle Diffusion coefficient, $D_{0}$ & $8.28 \cdot 10^{-12}\left[\mathrm{~m}^{2} / \mathrm{s}\right]$ \\
\hline Exponent $n$ (in Equation 8$)$ & 5 \\
\hline Parameter a (in Equation 8$)$ & $0.23 \cdot P e^{0.8}$ \\
\hline Parameter b (in Equation 8$)$ & $0.023 \cdot P e^{0.8}$ \\
\hline Applied magnetic field $H_{0}$ & $2.38 \cdot 10^{5}[\mathrm{~A} / \mathrm{m}]$ \\
\hline
\end{tabular}

Figure 5 instead shows the simulation of the trapping efficiency of the magnetic separation unit as a function of time (closed loop setup, long-term batch experiments). In this case, the model is capable of qualitatively capturing the experimental trends and also to provide a semi-quantitative prediction of the experimental data. The model always predicts that an increase in flow rate results in a decreased trapping efficiency of the unit.

The model is able to explain the different behavior of the two foams B and B'. While their overall total porosity is the same, the two foams have significantly different sizes of the tubular structures (here referred to as wires), with foam B having the largest size (Figure SI-3). A large diameter of the wires implies that the effect of convection is much enhanced, since the parameter that defines the relative role of convection is the product of the average convective velocity with the wire diameter. An increase of the diameter leads to much stronger hydrodynamic forces that the nanoparticles accumulated around a wire need to withstand, and this leads to a decrease in the maximum amount of nanoparticles that can be accumulated around each wire. The model is also able to account for the sharp decrease in trapping efficiency of all microreactors as the amount of nanoparticles loaded is doubled.

Nevertheless, some of the trends found for foam B' cannot be accounted for by the model in a quantitative manner. This is most probably due to the smaller pores in foam B' getting blocked by the NPs. This is caused by the aggregation of NPs in this pores induced by the initial high flow velocity. As the pores become blocked, the local velocity decreases, leading to even higher particles accumulation. This might explain the steadily increasing trapping efficiency in Figure 5c, which the model cannot account for.

We can anyhow conclude that the proposed model represents a useful tool to better understand the physics behind the separation process and can be used as a guideline for future design of the microreactor.

\section{Surface functionalization}

Slightly larger and better-defined APTES-SPIONs were used ${ }^{25}$ for the surface functionalization study. Figure 7 shows a model 2-step surface functionalization scheme of APTES-SPIONs.
In a first step, the surface of APTES-SPIONs was PEGylated by circulating the heterobifunctional Mal-PEG-NHS in the microreactor. PEG was chosen for several reasons: The surface functionalization of APTES-APIONS with PEG favors particle stabilization, as uncoated APTES-SPIONs are prone to aggregation at neutral $\mathrm{pH}$, in particular in the presence of electrolytes. PEG is by far the most widely used polymer in the field to impart steric stabilization (i) ${ }^{44}$. Moreover, the polymer and its homo- and hetero-bifunctional derivatives, respectively, are commercially available and allow for straightforward subsequent surface derivatization with e.g. fluorescent dyes or antibodies using standard bioconjugation techniques (ii). Currently, the most common way to mask NPs from the mononuclear phagocytic system is surface PEGylation, with the NPs being coated by the polymer, imparting stealth characteristics to inhibit plasma protein adsorption and recognition by the immune system (iii). ${ }^{44}$

The conjugation via the N-hydroxysuccinimidyl ester (NHS) reaction with the amine groups consists is a well-established method and leads to the formation of an amide bond. ${ }^{45}$

As expected, the initial zeta potential of $19.2 \mathrm{mV}$ (at $\mathrm{pH} 7.5$ ) for APTES-SPIONs decreased significantly after PEGylation to $-13.3 \mathrm{mV}$, which is in agreement with previous studies ${ }^{2,45}$. In parallel, we observed an increase of the mean particle hydrodynamic size from $63.3 \mathrm{~nm}$ (APTES-SPIONs) to $78.3 \mathrm{~nm}$ upon PEGylation. This increase of size can be explained by the presence of the MW 5000 PEG layer. ${ }^{2,45}$

In addition, we assessed successful PEG grafting by coupling a dye-labeled PEG in a parallel experiment to the APTESSPIONs. Any unreacted polymer was removed from the microreactor by extensive washing after the coupling reaction. The fluorescence spectra clearly showed an emission at 515 $\mathrm{nm}$, which is characteristic for the used dye (SI-5). From the fluorimetric assay we estimated a PEG grafting density of approximately 200 PEG molecules per particle, corresponding to a coverage density of 0.01 PEG chains per $\mathrm{nm}^{2}$ which is consistent with previous study. ${ }^{46}$

In a second step, the described PEG-APTES-SPIONs were further functionalized with cRGD peptide using heterobifunctional PEG as a crosslinker. The arginine-glycineaspartic acid (RGD) sequence plays a central role in cell recognition and many of the currently known integrins recognize this three amino acid sequence in their ligands ${ }^{47}$. Therefore, the deprotected cRGD peptide was labeled with an AlexaFluor 488 probe prior to coupling via the maleimide endterminated PEG-APTES-SPIONs in a separate microreactor experiment (figure 7). The mean size of cRGD-PEG-SPIONs increased from $78.3 \mathrm{~nm}$ to $83.9 \mathrm{~nm}$, which is consistent with previous studies. ${ }^{2}$ The zeta potential strongly increased from $13.3 \mathrm{mV}$ to $10.1 \mathrm{mV}$, thus indicating the presence of the peptide on the PEGylated NPs. Again, the fluorescent tag of the peptide allowed for detection and estimation of cRGD coupling, as described above. On average, roughly 20 cRGD molecules were bound per PEG-APTES-SPIONs.

PEGylation or cRGD coupling performed in a standard liquid phase synthesis always resulted in aggregation and low reaction 
yield, which is in agreement with previous studies from our group using large-scale magnetic microreactors. ${ }^{26}$

\section{Conclusions}

In this study we describe the design and development of magnetic microreactors for fast and efficient surface functionalization of superparamagnetic nanoparticles. Apart from extensive particle characterization, we focus on trapping and elution efficiency studies for different magnetic set-ups. In particular, we show how flow rate, elution time and reactor type impact trapping efficiency. We could also determine the maximum amount of SPIONs, which can be trapped within the microreactors, irrespective of their set-up. For the first time, the alignment of ultrasmall magnetic nanoparticles in a magnetic microreactor could be visualized with transmission electron microscopy. Finally, we propose a computational model of the trapping efficiency of the magnetic separation unit as a function of time, which agrees well with the experimental data. The use of magnetic microreactors opens new avenues not only for surface functionalization of SPIONs, but also for magnetic separation, up-concentration, sensing systems, or as nanocatalytic systems. The here described multifunctional NPs have been used in an in-depth microscopy study, where we use laser scanning (LSM), transmission electron (TEM) and dark field microscopy to investigate NP internalization and fate with macrophage (J774A.1) and epithelial (HeLa) cells in much detail. $^{48}$

\section{Acknowledgements}

This work was supported by the Swiss National Foundation (PP00P2-123373/1), the European Commission (7th Framework Program for Research 'Nanosciences, Nanotechnologies, Materials and New Production Technologies'; Grant number: NMP4-LA-2009-228929), the Adolphe Merkle Foundation and the University of Fribourg. The authors acknowledge Mr. Helmut Gnägi (Diatome AG, Biel, Switzerland) for expert help in ultramicrotomy. The support of the Dr. Alfred Bretscher Fund is gratefully acknowledged, and access to TEM was kindly provided by the Microscopy Imaging Centre of the University of Bern.

\section{Notes}

${ }^{a}$ Adolphe Merkle Institute, University of Fribourg, Route de l'ancienne papeterie CP 209, CH-1723 Marly 1, Switzerland.

${ }^{b}$ Chemistry Department, University of Fribourg, Chemin du musée 9, CH-1700 Fribourg, Switzerland.

${ }^{c}$ CSEM Alpnach, Centre Suisse d'Electronique et de Microtechnique, Untere Gründlistrasse 1, CH-6055 Alpnach Dorf, Switzerland.

$\dagger$ Electronic Supplementary Information (ESI) available: [TEM images of the magnetic alignment of particles inside the microreactor; light microscopy image of the nickel mesh and foams used in the microreactor; pictures of the different elements of the microreactor chamber; numerical simulation, trapping efficiency of each microreactor version]. See DOI: 10.1039/b000000x/

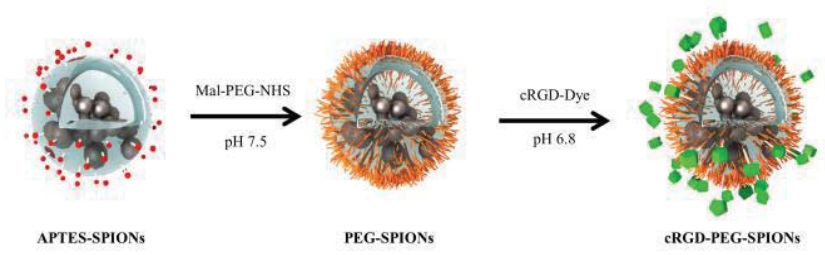

Figure 7: Schematic illustration of the multistep functionalization of APTESSPIONs with PEG and CRGD.

\section{References}

1. A. K. Gupta and M. Gupta, Biomaterials, 2005, 26, 3995-4021.

2. J. Salaklang, B. Steitz, A. Finka, C. P. O'Neil, M. Moniatte, A. J. van der Vlies, T. D. Giorgio, H. Hofmann, J. A. Hubbell, and A. PetriFink, Angew. Chem., Int. Ed., 2008, 47, 7857-7860.

3. T. Neuberger, B. Schöpf, H. Hofmann, M. Hofmann, and B. Rechenberg, J. Magn. Magn. Mater., 2005, 293, 483-496.

4. A.-H. Lu, E. L. Salabas, and F. Schüth, Angew. Chem., Int. Ed., 2006, 46, 1222-1244.

5. J. B. Haun, N. K. Devaraj, S. A. Hilderbrand, H. Lee, and R. Weissleder, Nat. Nanotechnol., 2010, 5, 660-665.

6. H. Häkkinen, Nat. Chem., 2012, 4, 443-455.

7. H. P. Khng, D. Cunliffe, S. Davies, N. A. Turner, and E. N. Vulfson, Biotechnol. Bioeng., 1998, 60, 419-424.

8. N. Nitin, L. E. W. LaConte, O. Zurkiya, X. Hu, and G. Bao, J. Biol. Inorg. Chem., 2004, 9, 706-712.

9. C. Zhang, B. Wängler, B. Morgenstern, H. Zentgraf, M. Eisenhut, H. Untenecker, R. Krüger, R. Huss, C. Seliger, W. Semmler, and F. Kiessling, Langmuir, 2007, 23, 1427-1434.

10. S. L. Ho, L. Jian, W. Gong, and W. N. Fu, IEEE Trans. Magn., 2012, 48, 3262-3265.

11. C. Alexiou, R. J. Schmid, R. Jurgons, M. Kremer, G. Wanner, C. Bergemann, E. Huenges, T. Nawroth, W. Arnold, and F. g. Parak, Eur. biophys. J., 2006, 35, 446-450.

12. M. M. Mojtahedi, M. S. Abaee, A. Rajabi, P. Mahmoodi, and S. Bagherpoor, J. Mol. Catal. A: Chem., 2012, 361-362, 68-71.

13. L. H. Reddy, J. L. Arias, J. Nicolas, and P. Couvreur, Chem. Rev., 2012, 112, 5818-5878.

14. C. T. Yavuz, J. T. Mayo, W. W. Yu, A. Prakash, J. C. Falkner, S. Yean, L. Cong, H. J. Shipley, A. Kan, M. Tomson, D. Natelson, and V. L. Colvin, Science, 2006, 314, 964-967.

15. C. R. Boehm, P. S. Freemont, and O. Ces, Lab Chip, 2013, 13, 3426.

16. M. Miyazaki, T. Honda, H. Yamaguchi, M. P. P. Briones, and H. Maeda, Biotechnol. Gen. Eng. Rev., 2008, 25, 405-428.

17. M. Medina-Sánchez, S. Miserere, and A. Merkoçi, Lab Chip, 2012, 12, 1932.

18. G. Bertotti, Hysteresis in magnetism: for physicists, materials scientists, and engineers, 1998.

19. I. M. Cohen and P. K. Kundu, Fluid mechanics, Academic Press, 2011. 
20. Z. Stekly, D. Fletcher, and J. Minervini, IEEE Trans. Magn., 1991, 27, 3655-3677.

21. M. Chastellain, A. Petri-Fink, and H. Hofmann, J. Colloid Interface Sci., 2004, 278, 353-360.

22. A. Bee, R. Massart, and S. Neveu, J. Magn. Magn. Mater., 1995, 149, 6-9.

23. G. A. van Ewijk, G. J. Vroege, and A. P. Philipse, J. Magn. Magn. Mater., 1999, 201, 31-33.

24. M. Yamaura, R. L. Camilo, L. C. Sampaio, M. A. Macêdo, M. Nakamura, and H. E. Toma, J. Magn. Magn. Mater., 2004, 279, 210217.

25. R. Digigow, J.-F. Dechézelles, H. Dietsch, I. Geissbühler, D. Vanhecke, C. Geers, A. Hirt, B. Rothen-Rutishauser, and A. PetriFink. Submitted.

26. B. Steitz, J. Salaklang, A. Finka, C. O'Neil, H. Hofmann, and A. Petri-Fink, Bioconjugate Chem., 2007, 18, 1-7.

27. S. Mohapatra, N. Pramanik, and S. Mukherjee, S. K. Ghosh, and P. Pramanik, J. Mater. Sci., 2007, 42, 7566-7574.

28. C. Vogt, M. S. Toprak, M. Muhammed, S. Laurent, J.-L. Bridot, and R. N. Müller, J. Nanopart. Res., 2009, 12, 1137-1147.

29. J. A. Ritter, A. D. Ebner, K. D. Daniel, and K. L. Stewart, J. Magn. Magn. Mater., 2004, 280, 184-201.

30. A. Figuerola, R. Di Corato, L. Manna, and T. Pellegrino, Pharmacol. Res., 2010, 62, 126-143.

31. R. W. Chantrell, A. Bradbury, and J. Popplewell, J. Appl. Phys., 1982, 53, 2742.

32. Q. Cao, X. Han, and L. Li, J. Phys. D: Appl. Phys., 2012, 45, 465001.

33. G. D. Moeser, K. A. Roach, W. H. Green, T. A. Hatton, and P. E. Laibinis, AIChE J., 2004, 50, 2835-2848.

34. Q. A. Pankhurst, J. Connolly, S. K. Jones, and J. Dobson, J. Phys. D: Appl. Phys., 2003, 36, 167-181.

35. R. Gerber, M. Takayasu, and F. J. Friedlaender, IEEE Trans. Magn., 1983, 19, 2115-2117.

36. M. Takayasu, R. Gerber, and F. J. Friedlaender, IEEE Trans. Magn., 1983, 19, 2112-2114.

37. T. Ying, S. Yiacoumi, and C. Tsouris, Chem. Eng. Sci., 2000, 55, 1101-1113.

38. A. Ditsch, S. Lindenmann, P. E. Laibinis, D. I. C. Wang, and T. A. Hatton, Ind. Eng. Chem. Res., 2005, 44, 6824-6836.

39. K. Hournkumnuard and C. Chantrapornchai, Simulation Modelling Practice and Theory, 2011, 19, 847-871.

40. F. Chen, K. A. Smith, and T. A. Hatton, AIChE J., 2012, 58, $2865-$ 2874.

41. M. Lattuada and M. Morbidelli, J. Colloid Interface Sci., 2011, 355, $42-53$.

42. R. Piazza, S. Buzzaccaro, and E. Secchi, J. Phys.: Condens. Matter, 2012, 24, 284109.

43. D. Y. C. Chan, D. Henderson, J. Barojas, and A. M. Homola, IBM J. Res. Dev., 1985, 29, 11-17.

44. D. E. Owens III and N. A. Peppas, Int. J. Pharm., 2006, 307, 93-102.

45. K. Herve, L. Douziech-Eyrolles, E. Munnier, S. Cohen-Jonathan, M. Soucé, H. Marchais, P. Limette, F. Warmont, M.-L. Saboungi, P. Dubois, I. Chourpa, Nanotechnology, 2008, 19, 465608.

46. X. Xia, M. Yang, Y. Wang, Y. Zheng, Q. Li, J. Chen, and Y. Xia, ACS Nano, 2012, 6, 512-522.
47. E. Ruoslahti, Annual Review of Cell and Developmental Biology, 1985, 29, 11-17.

48. R. G. Digigow, D. Vanhecke, B. Rothen-Rutishauser, M. J. D. Clift, and A. Petri-Fink, To be submitted. 